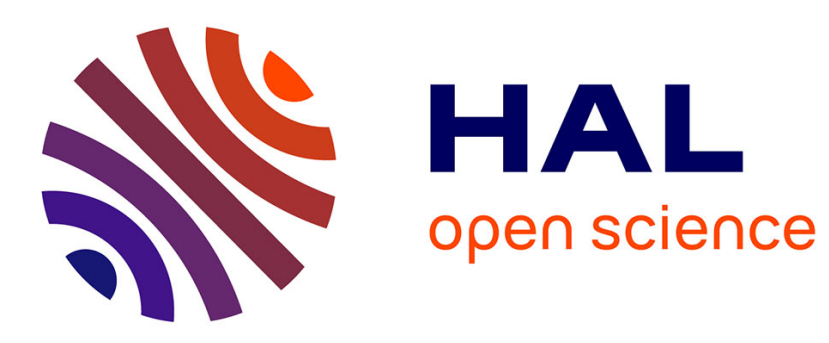

\title{
On Reconfigurability for Actuator Faults under Reliability Constraints
}

Ahmed Khelassi, Didier Theilliol, Philippe Weber

\section{To cite this version:}

Ahmed Khelassi, Didier Theilliol, Philippe Weber. On Reconfigurability for Actuator Faults under Reliability Constraints. Workshop on Automation in Mining, Mineral and Metal Industry, IFACMMM-2009, Oct 2009, Viña del Mar, Chile. pp.CDROM. hal-00530564

\section{HAL Id: hal-00530564 https://hal.science/hal-00530564}

Submitted on 29 Oct 2010

HAL is a multi-disciplinary open access archive for the deposit and dissemination of scientific research documents, whether they are published or not. The documents may come from teaching and research institutions in France or abroad, or from public or private research centers.
L'archive ouverte pluridisciplinaire HAL, est destinée au dépôt et à la diffusion de documents scientifiques de niveau recherche, publiés ou non, émanant des établissements d'enseignement et de recherche français ou étrangers, des laboratoires publics ou privés. 


\title{
On Reconfigurability for Actuator Faults under Reliability Constraints
}

\author{
A. Khelassi, D. Theilliol, P. Weber \\ Centre de Recherche en Automatique de Nancy (CRAN) \\ CNRS UMR 7039, Nancy-Université \\ BP 70239, 54506 Vandoeuvre Cedex, France \\ (Tel: +33383684 465; e-mail: ahmed.khelassi@cran.uhp-nancy.fr)
}

\begin{abstract}
This paper interests to the properties of fault-tolerant control system with respect to reliability of components. The aim of this paper is to present the need of reliability analysis in fault-tolerant control design. The focus of our study is on the reconfigurability analysis of systems with actuator faults under reliability constraints. This analysis proves that control reconfigurability is a system property that is not only related to the Gramian controllability and controllability of the system but it's related also and directly to overall system reliability. In this context, reconfigurability index is proposed. A flotation circuit is taking like an example to illustrate this work. In fact, flotation circuit is one of the most interactive mineral's processing operation. The interactive nature of flotation circuit results in oscillation of levels and hence of grades and recoveries.
\end{abstract}

Keywords: Fault-tolerant control system, Reconfigurability, Reliability, Actuator faults.

\section{INTRODUCTION}

Manufacturing systems consist of many different components which ensure their operation and high-quality production. In order to respect the growing of economic demand for high plant availability, and system safety, dependability is becoming an essential need in industrial automation. In this context and to satisfy these requirements, fault-tolerant control (FTC) is introduced. The aim of FTC systems is to keep plant available by the ability to achieve the objectives that have been assigned to the system in the faulty behavior and accept reduced performance when critical faults occur (Blanke et al. [2006], Zhang and Jiang [2008]). Thus, increasing systems autonomy involves the capability of critical system safety to compensate the impact of components fault on the system behavior. Within this framework, the main goal of FTC is to improve system's reliability which is rarely associated with an objective criterion that guides a design ( $\mathrm{Wu}$ [2001]). However, it is difficult to establish a functional linkage between the overall system reliability and control performance requirement.

In fault-tolerant control, the information obtained from the fault diagnosis module is used in the controller redesign. In fact, process diagnosis should not only indicate the fault occurrence but also identify the fault location and magnitudes. This assumption will make it possible the controller re-design. In this context, fault accommodation can be a mean to maintain the overall system operational after fault occurrence by adapting the controller parameters, or by generation of an additional control law (Blanke et al. [2001]). Moreover, if fault accommodation cannot be achieved, the complete control loop has to be reconfigured. Then, a new control law has to be designed and the controller structure has to be changed on-line. In this case, the original control objective is achieved, although degraded performance can be accepted.

Even though, it's crucial to study the property of the system and to determine which failure modes could severely affect the plant safety. However, only few attempts are now focusing on the fundamental FTC property analysis, some of studies are often defined as the fault detectability, fault isolability (Patton [1997]). In this context, the notion of reconfigurability was introduced as the control system quality under given faulty condition. In fact, first introduced in Moore [1981], the second order mode has been proposed as reconfigurability measures in Wu et al. [2000]. The reconfigurability of LTI system can be also evaluated using the controllability and observability Gramians (Frei et al. [1999], Wu and Bush [2004]). Moreover, a reconfigurability measures have been proposed to evaluate the size of fault-tolerant situations set named number of recoverable failures, under possible energy limitations (Staroswiecki [2002]), and also as general quadratic control problem (Staroswiecki [2003]). As shown in Yang [2006], the reconfigurability measures can be viewed like an intrinsic reconfigurability property or as a reconfigurability property performance. All these approaches have been proposing off-line. As recently considered by GonzalezContreras et al. [2009], the reconfigurability analysis can be evaluated on-line using input/output data.

The aim of this paper is to study the fault tolerance property in the presence of actuator faults. Our interest is to improve the availability and safety of system after fault occurrence to achieve the functional objectives until the end of the mission. In fact, before fault-tolerant control design, it is very important to ensure the commandability, the observability and the capability of a new control law to recover the faulty behavior with respect to the 
industrial demand formulated as overall system reliability requirement. In this framework and based on energy consumption, reliability analysis is introduced to predefine the cases for which the novel reconfiguration can not ensure the functional operating until final time of mission.

This paper is organized as follows: Section 2 formulates a fault-tolerant control problem and defines its reconfigurability concept as a system intrinsic property. Reconfigurability statement is examined based on limitation of energy consumption when actuator faults occur. In Section 3 , the reliability is introduced and its evaluation according to actuator faults magnitude is proposed in order to reformulate the reconfigurability condition based on energy limitation under reliability constraint. In this context, an index for reconfigurability property is proposed to evaluate performance degradation in the faulty case. Section 4 is devoted to illustrate this analysis. A flotation circuit is considered to evaluate the reconfigurability index. The mathematical model of a flotation circuit is developed for two cells under cascade configuration. Finally, conclusion is given in the last section.

\section{CONTROL RECONFIGURABILITY EVALUATION}

\subsection{Actuator faulty system representation:}

Consider the system in fault-free case modeled by a linear state-space representation (1):

$$
\left\{\begin{array}{l}
\dot{x}(t)=A x(t)+B u(t) \\
y(t)=C x(t)
\end{array}\right.
$$

where the state vector $x(t) \in \Re^{n}$, the control vector $u(t) \in \Re^{m}$, the output vector $y(t) \in \Re^{r}$ and matrices $A \in \Re^{n \times n}, B \in \Re^{n \times m}, C \in \Re^{r \times n}$.

In the faulty case, when system behavior is affected by actuator faults, the plant properties are not affected, but the influence of the control law on the plant is interrupted or modified. For this study, loss of control effectiveness is considered (Wu et al. [2000], Zhang and Jiang [2008]), and system (1) can be represented in the faulty case as follows:

$$
\left\{\begin{array}{l}
\dot{x}(t)=A x(t)+B_{f} u_{f}(t) \\
y(t)=C x(t)
\end{array}\right.
$$

where $B_{f}=B\left(I_{m}-\Gamma\right)$ and $\Gamma=\operatorname{diag}\{\gamma\}$. In fact, $\gamma=$ $\left\{\gamma_{1}, \gamma_{2}, \ldots \gamma_{m}\right\}$ represents a set of loss effectiveness factors $\gamma_{i}$ which define the values of actuator fault magnitudes, with $\gamma_{i} \in\left[\begin{array}{ll}0 & 1\end{array}\right]$. Indeed, if $\gamma_{i}=0$ actuator $i$ is considered under normal operation. Nevertheless, when $0<\gamma_{i}<1$, a fault is considered to represent a partial loss of control effectiveness. It is specified that fault is defined as a deviation of system parameters and performance from the nominal situation to an undesired way, so that the faulty system is remains operational in degraded mode. However, when $\gamma_{i}=1$ failure is considered and the actuator is out of order. In fact, the notion of failure describes the inability of a system or a component to accomplish its function. The failure is considered like an irrecoverable event at the system level (Blanke et al. [2001]).

\subsection{Reconfigurability based on controllability Gramian}

The control reconfigurability can be discussed as the ability of the considered system to recover some admissible system performance taken into account fault occurrence under energy limitation constraints (Staroswiecki [2002]). Used in the work of Staroswieski, the controllability Gramian appears to be useful for the following meanings: (1) guarantee the controllability condition of the system proving the existence of a solution; (2) there exists at least one admissible solution with respect to some specific energy limitation in order to take the state of system from $x(0)=x_{0}$ where $x_{0} \in \Re^{n}$ to the origin state $x(\infty)=0$ and to guarantee the existence of a reasonable solution. In this approach, the required energy consumption in the faulty case allows the reconfigurability measures in term of performance degradation according to the impact of fault on system behaviour. Indeed, this problem amounts minimize the energy consumed by the system. The criterion used is represented as following:

$$
\mathcal{J}\left(x_{0}\right)=\int_{0}^{\infty}\|u(t)\|^{2} d t
$$

The solution of (3) can be evaluated as a maximum energy which might be required to transfer the system state from $x_{0}$ to the origin. It can be computed by the maximum eigenvalue $\Lambda$ of controllability Gramian as following:

$$
\mathcal{J}\left(x_{0}, \gamma\right)=\max _{\left\|x_{0}\right\|=1} \Lambda\left[W_{c}^{-1}(\gamma)\right]
$$

where, $W_{c}$ is the controllability Gramian of the system (2) defined as following:

$$
W_{c}(\gamma)=\int_{0}^{\infty} \mathrm{e}^{A t} B_{f}(\gamma) B_{f}^{T}(\gamma) \mathrm{e}^{A^{T} t} d t
$$

It is known that $W_{c}(\gamma)$ can be evaluated as the unique solution of the following Lyapunov equation:

$$
A W_{c}(\gamma)+W_{c}(\gamma) A^{T}=-B_{f}(\gamma) B_{f}^{T}(\gamma)
$$

Which $W_{c}(\gamma)$ invertible and positive matrix since $A$ is stable and the pair $\left(A, B_{f}(\gamma)\right)$ keeps controllable. As proposed by Staroswiecki [2002], the actuators are characterized by the maximum eigenvalue of $W_{c}^{-1}(\gamma)$ defined by (7) which is interpreted as the maximum energy required transferring the system state to the origin.

$$
\sigma(\gamma)=\max \Lambda\left(W_{c}^{-1}(\gamma)\right)
$$

where $\Lambda$ is the eigenvalue of matrix $W_{c}^{-1}(\gamma)$.

\subsection{Reconfigurability index based on energy consumption}

In this approach, the fault tolerance is evaluated by means of the energy cost of the worst situation in which the system is still controllable for an admissible solution. Although, as illustrated in Staroswiecki [2002], the energetic cost does not really measure the system reconfigurability, but it evaluates the quality of the solution when they 
exist. Indeed, the degraded mode operating process is evaluated according to the consumption of energy required to restore the performance after fault occurrence. As illustrated in Gonzalez-Contreras et al. [2009], an index of reconfigurability based on maximum energy consumed (8) is proposed by normalization as:

$$
\rho(\gamma)=\frac{\sigma(\gamma)-\sigma_{\min }}{\sigma_{\max }-\sigma_{\min }}
$$

where $\sigma_{\max }$ is the upper value of energy in the worst case which corresponds to the functional mode most degraded. $\sigma_{\min }$ is the smallest value corresponding to the nominal case for $\gamma=0$. Due to the normalization of reconfigurability measure (7), index (8) values vary between 0 and $100 \%$. The index (9) can be seen like an image on the degradation of operation and system behaviour in term of performance according to the value of energy consumption in the degraded mode.

In order to establish limits of system reconfigurability in term of the energy consumed and acceptable degraded performance, admissibility is considered (Staroswiecki [2002], Gonzalez-Contreras et al. [2009]).

Definition 1. A solution for the problem of system control in the faulty case is admissible with respect to a control objective if:

$$
\rho(\gamma) \leq \rho_{\text {threshold }}
$$

where $\rho_{\text {threshold }}$ is a predefined threshold which represents the maximal functional degradation that can be admitted and accepted when a control solution is used.

As illustrated in Gonzalez-Contreras et al. [2009], the admissible values for the solution are established in order to limit the worst system operation in term of fault occurrence and its magnitude. The value $\rho_{\text {threshold }}$ can be specified off-line or on line. The latter depends on the evaluation of controllability Gramian from input/output data provided by the actual system measures.

\section{CONTROL RECONFIGURABILITY UNDER RELIABILITY CONSTRAINTS}

As presented previously, the reconfigurability based on the controllability Gramian is applied to evaluate the quality of the control which can be achieved by a fault-tolerant control scheme. However, to choose the reconfiguration strategy, it is very important to ensure that mean operating time of the novel configuration selected is sufficient to achieve the system's function until the end of the mission. This need involves the introduction of a specified condition in term of overall system reliability for the acceptable degraded mode through the estimation of fault effects on reliability proprieties. In this context, the problem of minimization (3) becomes a problem of energy minimization under industrial reliability constraint such as:

$$
\begin{aligned}
& J\left(x_{0}\right)=\int_{0}^{\infty}\|u(t)\|^{2} d t \\
& \text { s.t. } \quad R(t) \geq R_{o p t}
\end{aligned}
$$

where $R(t)$ is the overall system reliability. $R_{o p t}$ is a predefined threshold which represents the minimal value of the acceptable reliability in the degraded mode. In fact, reliability is the probability that units, components, equipments and systems will accomplish its intended function for a specified period of time under stated conditions and specific environments (Gertsbakh [2000]).

\subsection{Reliability computation}

In many situations and especially in the considered study, failure rates are obtained from components under different levels of loads.

Several mathematical models have been developed to define the failure level in order to estimate the failure rate $\lambda$ (Martorell et al. [1999]). Proportional hazard model introduced by Cox [1972] is used in this paper. The failure rate is modeled as follows:

$$
\lambda_{i}=\lambda_{i}^{0} \times g(\ell, \vartheta)
$$

where $\lambda_{i}^{0}$ represents the baseline failure rate (nominal failure rate) for the $i^{\text {th }}$ subsystem or component and $g(\ell, \vartheta)$ is a function (independent of time) taking into account the effects of applied loads with $\ell$ presenting an image of the load and $\vartheta$ defining some parameters of the subsystem or component. Different definitions of $g(\ell, \vartheta)$ exist in the literature. However, the exponential form is commonly used. Moreover, the failure rate functions for the exponential distribution change according to a load level. Under these remarks, the failure rate (11) is rewritten as:

$$
\lambda_{i}=\lambda_{i}^{0} \times \mathrm{e}^{\vartheta \times \ell}
$$

It can be noticed that load level (or mean load level) $\ell_{\gamma}$ is assumed to be directly associated to the control input of the actuator. As illustrated in Guenab et al. [2006], the value of failure rate change according to the intensity of control law on actuators due to fault occurrence. For the nominal behavior, the equation (12) can be written:

$$
\mathrm{e}^{\vartheta \times \ell}=\mathrm{e}^{\beta u_{\text {nom }}^{i}}
$$

where $\beta$ is a fixed coefficient and $u_{\text {nom }}^{i}$ is the nominal control law of the $i^{\text {th }}$ actuator in the fault-free case.

However, when even actuator fault occurs, loss of control effectiveness presented by $\gamma$ change and a new failure rate value is obtained due to the increasing of load level. In fact, in the context of FTC, the control law changes in order to recover the impact of fault on system performance. It can be established contingent on loss of control effectiveness $\gamma$ and $u_{\text {nom }}$ based on (1) and (2) as follows:

$$
u_{f}=(I-\Gamma)^{-1} u_{n o m}
$$

where $u_{f}$ is the control law delivered after the fault occurrence required to transfer the system state to the nominal case. Based on this aspect, failure rate can be modeled as follows: 


$$
\lambda_{i}(\gamma)=\lambda_{i}^{0} \times \mathrm{e}^{\left(1-\gamma_{i}\right)^{-1} u_{n o m}^{i}}
$$

Indeed, in degraded mode, overall system reliability decrease due to the evolution of the failure rate. It can be calculated in term of control effectiveness as follows:

$$
R_{i}(t, \gamma)=e^{-\lambda_{i}(\gamma) \times t}
$$

The reliability of overall system which is composed by different components or sub-systems depends on the way that these components are connected and on its reliabilities. In this context, for a system with $q$ series sub-systems, reliability is given by:

$$
R_{g}(t)=\prod_{i=1}^{q} R_{i}(t, \gamma)
$$

and with $q$ parallel sub-systems, is calculated as follows:

$$
R_{g}(t)=1-\prod_{i=1}^{q}\left(1-R_{i}(t, \gamma)\right)
$$

In general case, the system reliability is computed from a combination of the elementary function (17) and (18).

\subsection{Reconfigurability evaluation under reliability limitation constraints}

In order to improve fault tolerant control design, the measures of reconfigurability presented in section 2 is not adequate. Indeed, the system is considered reconfigurable if the availability of the novel configuration in degraded functional conditions is assured not only after fault occurrence but also until the mission final time. This condition is assumed for an admissible solution in term of energy consummation under reliability constraints. Indeed, The energy consumed gives an image on the performance degradation and the impact of actuator faults on system behavior. In this context, reliability is calculated a priori for all faulty possible situations defined by $\gamma$ at the end of mission $t_{m}$ as following:

$$
R_{i}\left(t_{m}, \gamma\right)=e^{-\lambda_{i}(\gamma) \times t_{m}}
$$

The degraded mode is defined by the value of $\gamma$ which represent the functional conditions of actuators. It can be characterized in term of functional performance quality by the overall system reliability at the end of mission defined by $R_{g}\left(t_{m}, \gamma\right)$. In fact, $R_{g}\left(t_{m}, \gamma\right)$ represents the lowest value of reliability which can be reached.

Indeed, for actuators loss effectiveness $\gamma=\{0,0, \cdots, 0\}$, the system is in the fault-free case and reliability at the last of mission is the upper value which can be reached.

$$
R_{g}\left(t_{m}, 0\right)=R_{\max }\left(t_{m}\right)
$$

However, for $\gamma=\{1,1, \cdots, 1\}$ overall system failure is considered, and the reliability at the end of mission $R_{g}\left(t_{m}, I\right)=0$. Moreover, when system is in degraded functional conditions, reliability can be evaluated as following:

$$
\forall \gamma_{i} \in\left[\begin{array}{ll}
0 & 1
\end{array}\right], \quad R_{g}\left(t_{m}, I\right) \leq R_{g}\left(t_{m}, \gamma\right) \leq R_{g}\left(t_{m}, 0\right)
$$

The property of reconfigurability is assured for an admissible solution of minimization problem (10) by condition (22).

$$
\gamma^{*}=\left\{\gamma \in \Re^{m},\left(\rho(\gamma) \leq \rho_{t}\right) \cup\left(R\left(t_{m}, \gamma\right) \geq R_{o p t}\right)\right\}
$$

The system is reconfigurable for acceptable degraded mode presented by the set of loss effectiveness control $\gamma^{*}$ which respects the requirements for control viewpoint and also for industrial reliability needs. The solution represents the acceptable degradation mode in term of control quality fixed by the index $\rho_{t}$ under industrial reliability constraints.

\section{EXAMPLE}

In the automation field, different works has interested on flotation process (Moys et al. [1992], Singh et al. [2003], Kampjarvi and Jamsa-Jounela [2003], Sbarbaro and Ortega [2005]). This section deals with the reconfigurability property of flotation circuit system is evaluated. Indeed, In the flotation process (Kampjarvi and JamsaJounela [2003]), the pulp is fed into the first cell and the froth is collected in the launders. The feed can be measured by a flow measurement, and the remaining pulp flows into the next cell. The magnitude of the flow depends on the pressure difference between two adjacent cells, the position of control valves, and the viscosity and density of the pulp. The magnitude of the pressure difference can be determined from the physical height difference of the cells. The pulp level in a cell is measured and controlled by adjusting the control valve. The following flotation circuit (Sbarbaro and Ortega [2005]) consists of cascade coupled tanks with control valves after each. All levels are measured and controlled by adjusting the flow rate through control valves and external inflow. Using mass balance equation associated with Torriceli rule, the system can be described by the following equations.

$$
\begin{gathered}
\frac{d V_{i}(t)}{d t}=q_{i n}(t)-C_{1}\left(u_{i}(t)\right) \sqrt{H_{1}(t)} \\
\frac{d V_{i}(t)}{d t}=C_{i-1}\left(u_{i-1}(t)\right) \sqrt{H_{i-1}(t)}-C_{i}\left(u_{i}(t)\right) \sqrt{H_{i}(t)}
\end{gathered}
$$

where $V$ is the liquid volume in the tank. the function $C_{i}$ represents the outflow rate of the $i^{t h}$ valve which depends directly on the input control signal $u_{i}(t) . H_{i}(t)$ is defined as:

$$
H_{i}(t)=2 g \times\left(h_{i}-h_{i-1}+\Delta h_{i}\right)
$$

where $g$ is the constant gravity acceleration and $\Delta h_{i}$ is the physical height difference between the $i^{t h}$ tank and the $i+1^{t h}$ excepted for the last one:

$$
H_{n}(t)=2 g \times\left(h_{n}+\Delta h_{n}\right)
$$


a non linear model for the valve, provided by valve manufacturer, is defined as:

$$
C_{i}\left(u_{i}(t)\right)=a_{1} d \times \frac{a_{2} u_{i}(t)+a_{2}\left(u_{i}(t)\right)^{2}}{1+e^{a_{4} u_{i}(t)}}
$$

with $d$ is the valve diameter and $a_{j}$ are constant parameters.

For this application, the process is composed of two valves and the control objective is to maintain the liquid level in the tanks in addition to industrial requirement of reliability. Time of actuator fault occurrence is supposed at $t=100 \mathrm{~min}$. The end of mission's time is fixed at: $t_{m}=200 \mathrm{~min}$. The following table presents the value of the nominal failure rates associated to the valves.

\section{Table 1. Failure rates of elementary components}

\begin{tabular}{|c|c|}
\hline \multicolumn{2}{|c|}{ Failure rates } \\
\hline$\lambda_{\text {valve } 1}$ & $2 \mathrm{e}-4$ \\
\hline$\lambda_{\text {valve } 2}$ & $5 \mathrm{e}-4$ \\
\hline
\end{tabular}

According to (27), a state space (1)describing the plant model, can be defined with the following matrices:

$$
A=\left[\begin{array}{ccc}
-0.4998 & 0.4998 & 0 \\
0.4998 & -0.7499 & 0.25 \\
0 & 0.25 & -0.75
\end{array}\right], \quad B=\left[\begin{array}{cc}
-1.3112 & 0 \\
1.3112 & -1.7438 \\
0 & 1.7438
\end{array}\right]
$$

and $C=I_{3}$.

Matrix $B$ is viewed as $B=\left[\begin{array}{ll}b_{1} & b_{2}\end{array}\right]$ and each of these vectors is affected by actuator faults as presented in equation (2). The controllability Gramian is calculated by using the Lyapunov equation (6) for different values of $\gamma_{i}$. In order to study the system reconfigurability, an index based on energy consumption is calculated for each degraded mode from (8) and the functional performance is evaluated from (9). This index is compared to a predefined threshold $\rho_{t}$ which represents the index of the acceptable functional degraded performance. In Fig.1, axis $x$ and $y$ show respectively the variation of $\left(\gamma_{1}, \gamma_{2}\right)$. Axis $z$ represents the evolution of index reconfigurability based on energy consumption. The energy is calculated from (8) for different degradation mode which defined according to different values of $\left(\gamma_{1}, \gamma_{2}\right)$ where $0 \leq \gamma_{1}<1$. The value of energy consumption increase according to the evolution of $\left(\gamma_{1}, \gamma_{2}\right)$ and the degradation performance is higher when value of actuators loss effectiveness is greater. It means that energy consumption defines the impact of fault intensity on system behavior and performance requirements. For this application, $\rho_{t}=20 \%$ defines the acceptable degradation of control performance.

In the other side, the overall system reconfigurability is evaluated from (17) where a flotation circuit is composed of two actuators embedded on series. The reliability is calculated for each degradation mode as follows:

$$
R_{g}\left(t_{m}\right)=R_{1}\left(t_{m}, \gamma_{1}\right) \cdot R_{2}\left(t_{m}, \gamma_{2}\right)
$$

To satisfy the industrial needs of reliability, it is necessary to ensure a reliability threshold predefined noted $R_{o p t}$. For this application, $R_{o p t}=75 \%$ which means that

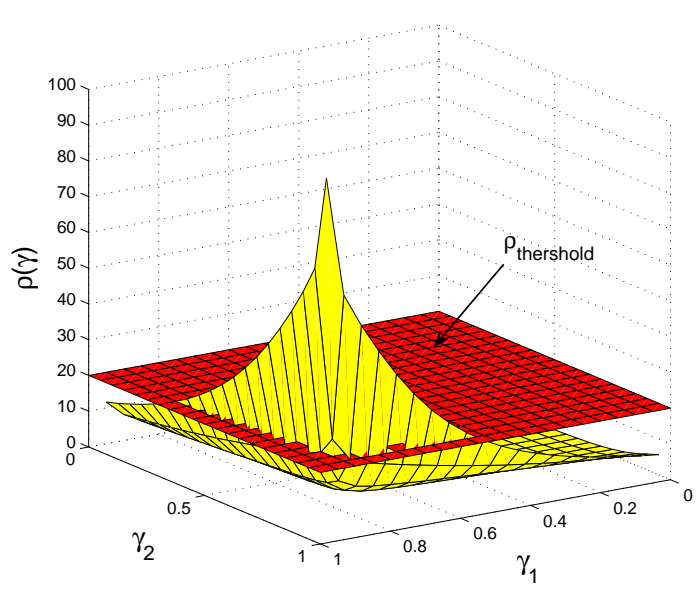

Fig. 1. Reconfiurabiliy index evolution based on energy consumption

for all degraded mode and after appearance of faults, the probability that a system accomplishes its intended function must be higher than $75 \%$. Taken into account this constraint, the reconfigurability is assured from (21). Fig.2 shows the overall system reliability evolution in degraded mode according to loss of control effectiveness, where axes $x$ and $y$ show respectively the variation of $\left(\gamma_{1}, \gamma_{2}\right)$.

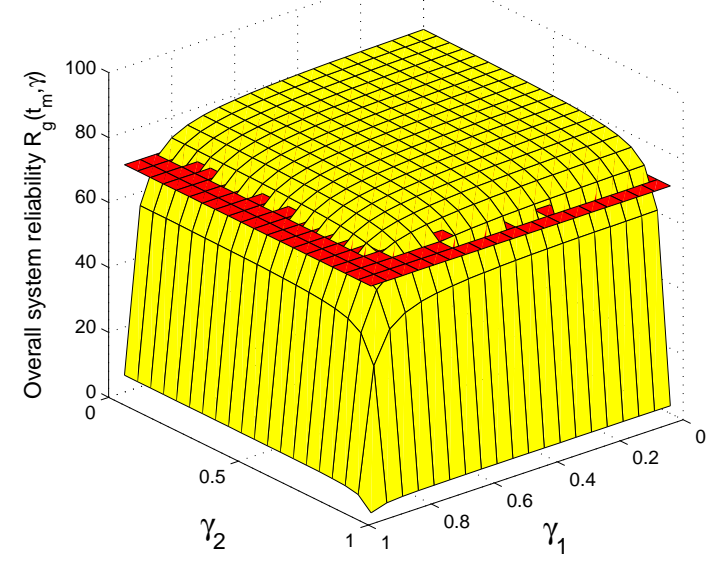

Fig. 2. Evolution of reliability in the end of mission in degraded mode

The acceptable degraded modes considered reconfigurable defined by (22) are presented in Fig.4, where axis $\mathrm{z}$ is an indicator of reconfigurability based on energy consumption under reliability constraints. Indeed, in Fig.3, the reconfigurability property is evaluated without reliability constraints

These results show the need of reliability evaluation in fault tolerant control design. In fact, as it can be showed, the evaluation of reconfigurability property based on energy consumption provides an indication on the probability to achieve the end of mission. 


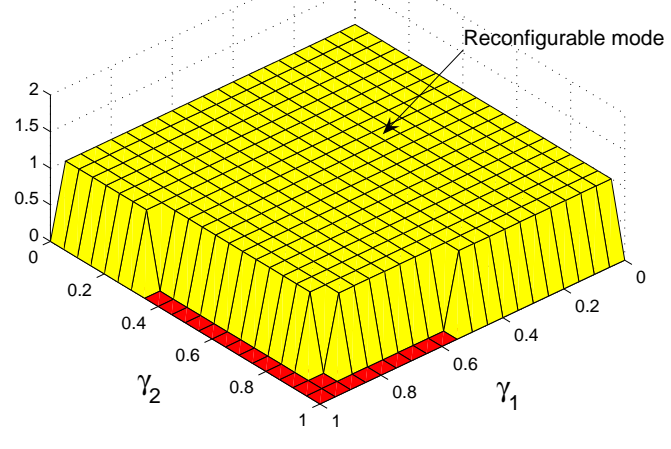

Fig. 3. Control Reconfigurability without reliability constraints

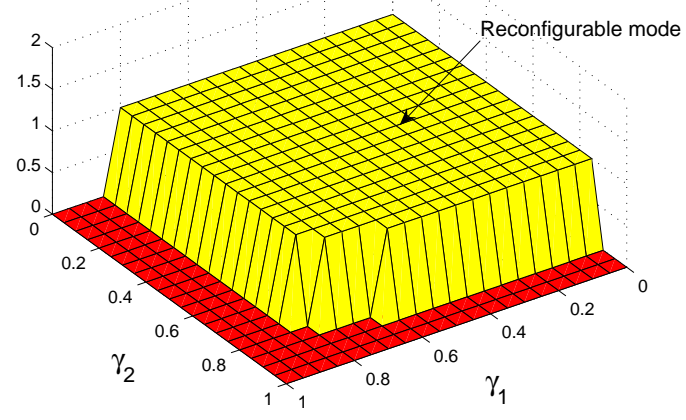

Fig. 4. Control Reconfigurability with reliability constraints

\section{CONCLUSION}

In this paper, a reconfigurability index based on the control reconfigurability according to reliability constraints has been proposed. Results obtained in our study prove that control reconfigurability is a system property that not only related to controllability Gramian and system controllability. It is shown that system reconfigurability is related also to overall system reliability. This relation characterizes those states reachable by an acceptable degraded functional and reliability needs. An illustrative example of a flotation circuit has been presented in order to highlight the ideas proposed.

\section{REFERENCES}

Blanke, M., Staroswiecki, M., and Wu, E.N. (2001). Concepts and methode in fault-tolerant control. American control conference, Proc. of 2001, volume 4, pages 26062620. Arlington, VA, USA.

Blanke, M., Kinnaert, M., Lunze, J., and Staroswiecki, M. (2006). Diagnosis and fault tolerant control. control Systems Series, Springer-Verlag London.

Cox, D.R. (1972). Regression models and life tables. JR Stat Soc, volume 34, pages 187-220.

Frei, C.W., Karus, F.J., and Blanke, M. (1999). Recoverability viewed as a system property. In Proc. of ECC99.
Gertsbakh, I. (2000). Reliability theory with applications to preventive maintenance. Springer.

Guenab. F., Theilliol, D., Weber, P., Zhang, Y.M., and Sauter, D. (2006). Fault tolerant control desing: A reconfiguration strategy based on reliability analysis under dynamic behaviour constraints. In Proc. of 6th IFAC symposium Safeprocess, Beijing, RP China.

Gonzalez-Contreras, B.M., Theilliol, D., and Sauter, D. (2009). On-line reconfigurability evaluation for actuator faults using input/output data. Accepted in IFAC SafeProcess'09.

Kampjarvi, P. and Jamsa-Jounela, S.L. (2003). Level control strategies for flotation cells. Minerals Engineering,volume 16, Issue 11, Pages 1061-1068.

Martorell, S., Sanchez, A., and Serradell, V. (1999). Agedependent reliability model considering effects of maintenance and working conditions. Reliability Engineering and System Safety, volume 64, pages 19-31.

Moore, B.C. (1981). Principal component analysis in linear systems: controllability observability and momdel reduction. IEEE Transaction on Automatic control, volume 26, pages 17-32.

Moys, M.H., Engelbrecht, J., and Terblanche, N. (1992). Further developments in the control of levels in flotation circuit. Journal of the South African Institute of Mining and Metallurgy,volume 92, Issue 2, Pages 39-45.

Patton, R.J. (1997). Fault-tolerant control; The 1997 situation. In Proc. IFAC SafeProcess'97, pages 10331055.

Sbarbaro, D. and Ortega, R. (2005). Averaging Level Control of Multiple Tanks: A Passivity Based Approach. 44th IEEE Conference on Decision and Control, CDCECC'05.

Singh, A., Louw, J.J., and Hulbert, D.G. (2003). Flotation stabilization and optimization. Journal of the South African Institute of Mining and Metallurgy,volume 103, Issue 9, Pages 581-588.

Staroswiecki, M. (2002). On reconfigurability with respect to actuator failures . 15th Terminal word congress of IFAC'2002, Barcelona, Spain.

Staroswiecki, M. (2003). Actuator faults and the linear quadratic control problem. In Proc. of the 42nd Conference on Decision and control, Maui, Hawwaii, USA.

$\mathrm{Wu}$, N.E., Zhou, K., and Salmon, G. (2000). Control reconfigurability of linear time-invariant systems. Elseiver science Ltd Automatica, pages 1767-1771.

Wu, N.E. (2001). Reliability and fault tolerant control system Part II. IEEE Conference on decision and control, Orlando, Florida, USA.

Wu, N.E. and Bush, T. (2004). Operational reconfigurability in command and control. In Proc. of the 2004 American control conference, Boston, Massachusetts, USA.

Yang, Z. (2006). Reconfigurability analysis for a class of linear hybrid systems. In Proc. of 6th IFAC Safeprocess'06, Beijing, PR china.

Zhang, Y. and Jiang, J. (2008). Bibliographical review on reconfigurable tolerant-control system. Annual Reviews in Control, volume 32, pages 229-252. 